\title{
GENERALIZATIONS TO SPACE OF THE CAUCHY AND MORERA THEOREMS $\left({ }^{1}\right)$
}

BY

\author{
MAXWELL READE AND E. F. BECKENBACH
}

Consider the function

\section{INTRODUCTION}

$$
w=f(z)=x_{1}(u, v)+i x_{2}(u, v),
$$$$
z=u+i v,
$$

defined and continuous in a simply connected domain $D\left({ }^{2}\right)$. A necessary and sufficient condition that $f(z)$ be analytic in $D$ is that the Cauchy-Riemann equations be satisfied there:

$$
\lambda w=0,
$$

where

$$
\lambda \equiv \frac{\partial}{\partial u}+i \frac{\partial}{\partial v}
$$

is a differential operator. From (2) we obtain

$$
\sum_{j=1}^{2}\left(\lambda x_{j}\right)^{2}=0
$$

According to the Cauchy and Morera theorems, a necessary and sufficient condition that the continuous function (1) be analytic in the simply connected domain $D$ is that for each closed rectifiable Jordan curve $\gamma$ lying in the domain $D$,

$$
\int_{\gamma} f(z) d z=0
$$

Now (4) may be considered to be an integral analogue of the differential condition (2); it implies

$$
\sum_{j=1}^{2}\left[\int_{\gamma} x_{j}(u, v) d z\right]^{2}=0,
$$

which is analogous to (3).

Presented to the Society April 14, 1939, under the title $A$ characterization of plane isothermic maps; received by the editors March 4, 1940.

(1) Some of the results of this paper have been summarized in the authors' note of the same title in the Proceedings of National Academy of Sciences, vol. 25 (1939), pp. 92-97. finite.

(2) It is to be understood throughout this paper that the domains under consideration are 
If (3) holds, then either $x_{1}+i x_{2}$ or $x_{2}+i x_{1}$ is an analytic function of $z=u+i v$, and $x_{1}(u, v)$ and $x_{2}(u, v)$ are said to form $a$ couple of conjugate harmonic functions. We note that (5) is a necessary and sufficient condition that the continuous functions $x_{1}(u, v)$ and $x_{2}(u, v)$ form a couple of conjugate harmonic functions.

The real functions

$$
x_{j}=x_{j}(u, v), \quad j=1,2,3,
$$

defined and continuous in a simply connected domain $D$, will be said to define a surface $S$. If the first partial derivatives of the functions (6) are continuous and satisfy

$$
E(u, v)=G(u, v), \quad F(u, v)=0
$$

in $D$, where

$$
E(u, v) \equiv \sum_{j=1}^{3}\left(\frac{\partial x_{j}}{\partial u}\right)^{2}, \quad F(u, v) \equiv \sum_{j=1}^{3} \frac{\partial x_{j}}{\partial u} \frac{\partial x_{j}}{\partial v}, \quad G(u, v) \equiv \sum_{j=1}^{3}\left(\frac{\partial x_{j}}{\partial v}\right)^{2}
$$

are the coefficients of the first fundamental differential quadratic form of the surface $S$, then the parameters $u, v$ are said to be isothermic parameters, and $S$ is said to be given in isothermic representation. The map of $D$ on $S$ is conformal except where $E=G=0$. From (8) it follows that (7), which is a generalization to space of (3), can be written in the form

$$
\sum_{j=1}^{3}\left(\lambda x_{j}\right)^{2}=0
$$

An analogous generalization to space of (5) is

$$
\sum_{j=1}^{3}\left[\int_{\gamma} x_{j}(u, v) d z\right]^{2}=0,
$$

where $\gamma$ is a closed rectifiable Jordan curve lying in $D$. In this paper we shall study (10) and

$$
\sum_{j=1}^{3}\left[\int_{C_{r}} x_{j}(u, v) d z\right]^{2}=o\left(r^{\alpha}\right),
$$

where $C_{r}$ is the circle in $D$ with center at the arbitrary point $\left(u_{0}, v_{0}\right)$ of $D$ and of radius $r$, and where $o\left(r^{\alpha}\right)$ denotes a quantity (not always the same quantity) such that

$$
\lim _{r \rightarrow 0} \frac{o\left(r^{\alpha}\right)}{r^{\alpha}}=0
$$

If the functions (6) are harmonic and satisfy (9) in a simply connected 
domain $D$, they have been called a triple of conjugate harmonic functions $\left({ }^{3}\right)$. In terms of this definition, a theorem of Weierstrass may be stated as follows.

$A$ necessary and sufficient condition that the functions (6), defined in a simply connected domain, be the coordinate functions of a minimal surface given in isothermic representation is that they form a triple of conjugate harmonic functions.

We shall have use for the following direct computation. Let the functions (6) have continuous partial derivatives of the $m$ th order in a simply connected domain $D$; then about each point $\left(u_{0}, v_{0}\right)$ of $D$ we have a finite Taylor expansion for each function:

$$
x_{j}(u, v)=\sum_{n=0}^{m} \frac{r^{n}}{n !}\left[\left(\cos \theta \frac{\partial}{\partial u}+\sin \theta \frac{\partial}{\partial v}\right)^{n} x_{j}\right]+o\left(r^{m}\right), \quad j=1,2,3,
$$

where

$$
\cos \theta \frac{\partial}{\partial u}+\sin \theta \frac{\partial}{\partial v}
$$

is a differential operator, where the partial derivatives are evaluated at the point $\left(u_{0}, v_{0}\right)$, and where $u-u_{0}=r \cos \theta, v-v_{0}=r \sin \theta$. Then, for the circle $C_{r}$ in $D$ with center at $\left(u_{0}, v_{0}\right)$ and radius $r$, the left-hand member of (10) assumes the form

$$
\sum_{j=1}^{3}\left[\int_{C_{r}} x_{j}(u, v) d z\right]^{2}=-\pi^{2} \sum_{k=0}^{[(m-1) / 2]} \sum_{p=0}^{k} r^{2 k+4} B_{p, k}+o\left(r^{m+3}\right),
$$

where

where

$$
B_{p, k} \equiv \sum_{j=1}^{3} \frac{C_{k+1, p+1} C_{k+1, p}}{2^{2 k}[(k+1) !]^{2}} \Delta^{p} \lambda x_{j} \Delta^{k-p} \lambda x_{j}
$$

$$
\Delta \equiv \lambda \bar{\lambda} \equiv \frac{\partial^{2}}{\partial u^{2}}+\frac{\partial^{2}}{\partial v^{2}}, \quad \Delta^{0} \lambda x_{j} \equiv \lambda x_{j}
$$

where the $C_{k, 8}$ are binomial coefficients, $C_{k, s} \equiv k ! / s !(k-s) !, C_{k, 0} \equiv 1$, and where $[(m-1) / 2]$ is the greatest integer not greater than $(m-1) / 2$.

For $m=1,3,5,(12)$ is displayed in (14), (48) and (35) respectively.

\section{Characterization of isothermic maps}

THEOREM 1. If the functions (6) have continuous partial derivatives of the first order in a simply connected domain $D$, then a necessary and sufficient condition that they map $D$ isothermically on a surface $S$ is that for each point $\left(u_{0}, v_{0}\right)$ of $D$,

(3) E. F. Beckenbach and T. Rado, Subharmonic functions and minimal surfaces, these Transactions, vol. 35 (1933), pp. 648-661. 


$$
\sum_{j=1}^{3}\left[\int_{C_{r}} x_{j}(u, v) d z\right]^{2}=o\left(r^{4}\right),
$$

where $C_{r}$ is the circle in $D$ with center at $\left(u_{0}, v_{0}\right)$ and radius $r$.

Proof. If the first partial derivatives of the functions (6) are continuous in $D$, then we obtain a finite Taylor expansion for each function about an arbitrary point $\left(u_{0}, v_{0}\right)$ of $D$ by setting $m=1$ in (11). If $C_{r}$ is the circle in $D$ with center at $\left(u_{0}, v_{0}\right)$ and radius $r$, then upon setting $m=1$ in (12) we obtain

$$
\sum_{j=1}^{3}\left[\int_{C_{r}} x_{j}(u, v) d z\right]^{2}=-\pi^{2} r^{4} \sum_{j=1}^{3}\left(\lambda x_{j}\right)^{2}+o\left(r^{4}\right) .
$$

From (14) it follows that a necessary and sufficient condition that the relation (9) hold is that (13) hold.

\section{ChaRACTERIZATION OF THOSE ISOTHERMIC SPHERICAL MAPS THAT DO NOT MAP CIRCLES ON CIRCLES}

LEMMA 1. If the functions (6) are defined, but not identically constant, in a simply connected domain $D$, and if they map $D$ isothermically on a surface $S$ that lies on a sphere $S$ of finite radius $r$, then a necessary and sufficient condition that they map circles on circles is that the first quadratic form of $S$ have the representation

$$
d s^{2}=\frac{c^{2}\left(d u^{2}+d v^{2}\right)}{\left[\left(u-u_{0}\right)^{2}+\left(v-v_{0}\right)^{2}+a^{2}\right]^{2}}, \quad a>0, c>0 .
$$

Necessity. Let the coordinates of the center of $S$ be denoted by $\left(a_{1}, a_{2}, a_{3}\right)$, and let an $s, t$-plane be so placed that the positive $s$-and $t$-axes coincide with the positive $x_{1}$ - and $x_{2}$-axes respectively. Let $S$ be projected stereographically on the $s, t$-plane, the coordinates of the pole of projection being $\left(a_{1}, a_{2}, k\right)$, where $k=a_{3} \pm r$ and $|k|$ is the maximum of the two quantities $\left|a_{3}+r\right|$ and $\left|a_{3}-r\right|$. We have

$$
\begin{aligned}
& x_{1}=a_{1}+\frac{2 r|k|\left(s-a_{1}\right)}{\left(s-a_{1}\right)^{2}+\left(t-a_{2}\right)^{2}+k^{2}}, \\
& x_{2}=a_{2}+\frac{2 r|k|\left(t-a_{2}\right)}{\left(s-a_{1}\right)^{2}+\left(t-a_{2}\right)^{2}+k^{2}}, \\
& x_{3}=k\left[1-\frac{2 r|k|}{\left(s-a_{1}\right)^{2}+\left(t-a_{2}\right)^{2}+k^{2}}\right] .
\end{aligned}
$$

Let the map of $S$ on the $s, t$-plane be $D^{\prime}$. The product of the transformation (6) and the stereographic projection maps $D$ on $D^{\prime}$, and carries circles in $D$ into circles in $D^{\prime}$. It follows that this isothermic map of $D$ on $D^{\prime}$ is equivalent 
to a single linear transformation:

$$
F(z)=s+i t=\frac{\alpha^{\prime} z+\beta^{\prime}}{\gamma^{\prime} z+\delta^{\prime}}
$$

or

$$
F(z)=s+i t=\frac{\alpha^{\prime} \bar{z}+\beta^{\prime}}{\gamma^{\prime} \bar{z}+\delta^{\prime}}, \quad \quad\left(\alpha^{\prime} \delta^{\prime}-\beta^{\prime} \gamma^{\prime}\right) \neq 0,
$$

where (a) holds if the map of $D$ on $D^{\prime}$ is directly conformal, and where (b) holds if the map is inversely conformal. From (16) and (17) it follows that the functions (6) have the form

$$
\begin{gathered}
x_{1}=a_{1}+\frac{2 r|k| R f(z)}{f(z) \overline{f(z)}+k^{2}}, \quad x_{2}=a_{2}+\frac{2 r|k| J f(z)}{f(z) \overline{f(z)}+k^{2}}, \\
x_{3}=k\left[1-\frac{2 r|k|}{f(z) \overline{f(z)}+k^{2}}\right]
\end{gathered}
$$

where

$$
f(z)=F(z)-\left(a_{1}+i a_{2}\right)
$$

and where $R f(z)$ is the real part, and $\exists f(z)$ the imaginary part, of $f(z)$.

There are two possible representations for the functions (6) as determined by (17), (18) and (19). It is now an easy matter to compute $E, F$ and $G$ and to show that the first quadratic form of $S$ has the representation $(15)\left({ }^{4}\right)$.

We note that if it is given only that one non-null circle in $D$ is mapped on a circle on $S$ by the isothermic functions (6), then, as in the above discussion, $D$ is mapped isothermically on $D^{\prime}$ such that one non-null circle in $D$ is mapped on a circle in $D^{\prime}$. Therefore the function mapping $D$ isothermically on $D^{\prime}$ is linear; and hence it follows that the functions (6) have the form (18) and map all circles in $D$ on circles on $S$.

Sufficiency. Let the stereographic projection of $D$ on the sphere $S^{\prime}$ be $S^{\prime}$, where $S^{\prime}$ is the sphere with center at $\left(u_{0}, v_{0}, a-c / 2 a\right)$ and radius $c / 2 a$, and where the pole of projection is at $\left(u_{0}, v_{0}, a\right)$. This projection is given by (18), where

$$
a_{1}=u_{0}, \quad a_{2}=v_{0}, \quad k=a, \quad r=c / 2 a, \quad f(z)=(u+i v)-\left(u_{0}+i v_{0}\right) .
$$

The first quadratic form of $S^{\prime}$ is found to be identical with that of $S$; hence $S^{\prime}$ and $S$ are applicable. Therefore $S^{\prime}$ and $S$ are either congruent or symmetric;

(4) We find $d s^{2}=E\left(d u^{2}+d v^{2}\right)=c^{2}\left(d u^{2}+d v^{2}\right) /\left[\left(u-u_{0}\right)^{2}+\left(v-v_{0}\right)^{2}+\eta\right]^{2}$. But then a computation shows that the Gaussian curvature, given by the formula in (47), which is positive on $S$, has the value $4 \eta / c^{2}$, so that $\eta=a^{2}$, where $a>0$. 
in either case since circles in $D$ are mapped on circles on $S^{\prime}$, it follows that circles in $D$ are mapped on circles on $S$.

THEOREM 2. If the functions (6) are defined, but not identically constant, in a simply connected domain $D$, and if they have continuous partial derivatives of the third order in $D$, then a necessary and sufficient condition that they map $D$ isothermically on a surface $S$ that lies on a sphere of finite radius, such that circles are not mapped on circles, is that

$$
\sum_{j=1}^{3}\left[\int_{C_{r}} x_{j}(u, v) d z\right]^{2}=o\left(r^{6}\right)
$$

hold for all points $\left(u_{0}, v_{0}\right)$ in $D$, while

$$
\sum_{j=1}^{3}\left[\int_{C_{r}} x_{j}(u, v) d z\right]^{2} \neq o\left(r^{8}\right)
$$

hold for some points $\left(u_{0}, v_{0}\right)$ in $D$, where $C_{r}$ is the circle in $D$ with center at $\left(u_{0}, v_{0}\right)$ and radius $r$.

Necessity. Under the hypotheses, the functions (6) map $D$ isothermically on a surface that lies on a sphere of finite non-null radius. Hence (9) holds, and there exists a constant $\alpha$ such that

$$
e=\alpha E, \quad f=\alpha F, \quad g=\alpha G,
$$

$\alpha \neq 0$,

where $e, f$ and $g$ are the coefficients of the second fundamental quadratic form of $S\left({ }^{5}\right)$.

For the present representation of $S$, the formulas of Gauss $\left(^{6}\right)$ become

$$
\begin{array}{cc}
x_{j, u u}=R_{u} x_{j, u}- & R_{v} x_{j, v}+e \zeta_{j}, \quad x_{j, u v}=R_{v} x_{j, u}+R_{u} x_{j, v}, \\
x_{j, v v}=-R_{u} x_{j, u}+R_{v} x_{j, v}+e \zeta_{j}, & j=1,2,3,
\end{array}
$$

where

$$
R \equiv \frac{1}{2} \log E,
$$

and where $\zeta_{j}, j=1,2,3$, are the direction cosines of the normal to $S$.

We shall need the following relations:

$$
\begin{gathered}
\sum_{j=1}^{3} \lambda x_{j} \Delta^{2} \lambda x_{j}=-4 \alpha^{2} E \lambda^{2} E, \\
\sum_{j=1}^{3}\left(\Delta \lambda x_{j}\right)^{2}=4 \alpha^{2}(\lambda E)^{2} .
\end{gathered}
$$

(5) For information concerning the second fundamental differential quadratic form of a surface, see W. C. Graustein, Differential Geometry; in particular, we have referred to pages 9394 and pages $97-98$.

(6) Graustein, op. cit., pp. 135-137. 
To obtain (24) and (25), we shall use a method which depends upon the existence and continuity of partial derivatives of order higher than three of the functions (6). These functions map $D$ isothermically on a spherical surface. Therefore, as in the proof of Lemma 1, we can consider an intermediate stereographic projection to show that the functions (6) have the representation (18), where either $f(z)$ or $f(\bar{z})$ is analytic in $D$. From this representation, it follows that the functions (6) have continuous partial derivatives of all orders.

To obtain (24) and (25) we shall need the following equalities:

$$
\begin{gathered}
\sum_{j=1}^{3} \zeta_{j} \lambda x_{j}=0, \\
\sum_{j=1}^{3} \lambda x_{j} \lambda \zeta_{j}=0, \\
\sum_{j=1}^{3} \lambda^{2} \zeta_{j} \lambda x_{j}=0, \\
\sum_{j=1}^{3} \lambda x_{j} \Delta \zeta_{j}=0, \\
\sum_{j=1}^{3} \lambda x_{j} \Delta \lambda \zeta_{j}=0 .
\end{gathered}
$$

$$
j=1,2,3,
$$

$$
\begin{aligned}
\Delta^{2} \lambda x_{j}=2\left(e \Delta \lambda \zeta_{j}+2 \lambda e \Delta \zeta_{j}+2 \Delta e \lambda \zeta_{j}+\bar{\lambda} e \lambda^{2} \zeta_{j}+\lambda^{2} e \bar{\lambda} \zeta_{j}+\zeta_{j} \Delta \lambda e\right), & j=1,2,3,
\end{aligned}
$$

It follows from (23) that

$$
\Delta \lambda x_{j}=2 \lambda\left(e \zeta_{j}\right),
$$$$
j=1,2,3,
$$

and hence (26) holds. Operating on (26) with the operator $\bar{\lambda}$, and then operating on this result with $\lambda$, we obtain (27).

The formulas of Olinde Rodriguez $\left(^{7}\right)$ may be written together in the following form :

$$
\lambda \zeta_{j}=-\alpha \lambda x_{j}, \quad \alpha \neq 0, j=1,2,3 .
$$

From (33) we obtain

$$
\sum_{j=1}^{3} \lambda x_{j} \lambda \zeta_{j}=-\alpha \sum_{j=1}^{3}\left(\lambda x_{j}\right)^{2},
$$

which, with (9), yields (29).

From (29) we obtain

(?) Graustein, op. cit., p. 121. 


$$
\lambda \sum_{j=1}^{3} \lambda \zeta_{j} \lambda x_{j}=\sum_{j=1}^{3} \lambda^{2} \zeta_{j} \lambda x_{j}+\sum_{j=1}^{3} \lambda \zeta_{j} \lambda^{2} x_{j}=0,
$$

which, with (33), establishes (30).

From (29) we obtain

$$
\bar{\lambda} \sum_{j=1}^{3} \lambda \zeta_{j} \lambda x_{j}=\sum_{j=1}^{3} \Delta \zeta_{j} \lambda x_{j}+\sum_{j=1}^{3} \lambda \zeta_{j} \Delta x_{j}=0,
$$

which, with (33), establishes (31).

From (26), (28) and (29) we obtain

$$
\sum_{j=1}^{3} \lambda x_{j} \Delta \lambda x_{j}=0
$$

which, with (33), yields (32).

From (27)-(32), inclusive, it follows that

$$
\sum_{j=1}^{3} \lambda x_{j} \Delta^{2} \lambda x_{j}=2 \lambda^{2} e \sum_{j=1}^{3} \lambda x_{j} \bar{\lambda} \zeta_{j}
$$

From (8), (9), (22), (33) and (34) we obtain (24).

From (9) and (33) we obtain

$$
\sum_{j=1}^{3}\left(\lambda \zeta_{j}\right)^{2}=0
$$

which combines with (22), (26) and (33) to yield (25).

Since the functions (6) have continuous partial derivatives of all orders in $D$, the following expression, obtained from (12) by setting $m=5$, is valid:

$$
\begin{aligned}
\sum_{j=1}^{3}\left[\int_{C_{r}} x_{j}(u, v) d z\right]^{2}= & -\pi^{2} r^{4} \sum_{j=1}^{3}\left(\lambda x_{j}\right)^{2}-\frac{\pi^{2} r^{6}}{4} \sum_{j=1}^{3} \lambda x_{j} \Delta \lambda x_{j} \\
& -\frac{\pi^{2} r^{8}}{192} \sum_{j=1}^{3}\left[2 \lambda x_{j} \Delta^{2} \lambda x_{j}+3\left(\Delta \lambda x_{j}\right)^{2}\right]+o\left(r^{8}\right) .
\end{aligned}
$$

Here the partial derivatives are evaluated at the point $\left(u_{0}, v_{0}\right)$ which is the center of the arbitrary circle $C_{r}$ in $D$. Applying (9) and the above relation $\sum_{j=1}^{3} \lambda x_{j} \Delta \lambda x_{j}=0$, to (35), we obtain

$$
\sum_{j=1}^{3}\left[\int_{C_{r}} x_{j}(u, v) d z\right]^{2}=-\frac{\pi^{2} r^{8}}{192} \sum_{j=1}^{3}\left[2 \lambda x_{j} \Delta^{2} \lambda x_{j}+3\left(\Delta \lambda x_{j}\right)^{2}\right]+o\left(r^{8}\right) .
$$

Therefore (20) holds. in $D$

Let us suppose that (21) does not hold, i.e., that for each point $\left(u_{0}, v_{0}\right)$ 


$$
\sum_{j=1}^{3}\left[\int_{C_{r}} x_{j}(u, v) d z\right]^{2}=o\left(r^{8}\right),
$$

where $C_{r}$ is the circle in $D$ with center at $\left(u_{0}, v_{0}\right)$ and radius $r$. From (36) and (37) it follows that

$$
\sum_{j=1}^{3}\left[2 \lambda x_{j} \Delta^{2} \lambda x_{j}+3\left(\Delta \lambda x_{j}\right)^{2}\right]=0 .
$$

From (24), (25) and (38) we obtain

$$
\alpha^{2}\left[2 E \lambda^{2} E-3(\lambda E)^{2}\right]=0 .
$$

But, by (22), $\alpha \neq 0$, and therefore (39) yields

$$
2 E \lambda^{2} E-3(\lambda E)^{2}=0 .
$$

From (40) we obtain

$$
2 \lambda \log (\lambda E)=3 \lambda(\log E)
$$

therefore

$$
\lambda E=E^{3 / 2} e^{Q(2)},
$$

where

$$
\lambda Q(z)=0 .
$$

From the imaginary part of $(40)$ we obtain

$$
2 \frac{E_{u v}}{E_{u}} d v=3 \frac{E_{v}}{E} d v, \quad 2 \frac{E_{u v}}{E_{v}} d u=3 \frac{E_{u}}{E} d u,
$$

which imply

$$
E_{u}=E^{3 / 2} e^{\Phi 1(u)} \quad E_{v}=E^{3 / 2} e^{\Phi 2(v)}
$$

where $\Phi_{1}(u)$ is a function of $u$ alone and $\Phi_{2}(v)$ is a function of $v$ alone. From (41), (42) and (43) it follows that the function

$$
e^{Q(z)} \equiv e^{\Phi 1(u)}+i e^{\Phi_{2}(v)}
$$

is an analytic function of $z=u+i v$. From the Cauchy-Riemann equations for the function (44) it follows that

$$
e^{\Phi_{1}(u)} \equiv 2 a_{0} u+a_{1}, \quad e^{\Phi_{2}(v)} \equiv 2 a_{0} v+a_{2},
$$

where $a_{0}, a_{1}$ and $a_{2}$ are real constants, and hence that (41) yields

$$
\frac{E_{u}}{E^{3 / 2}} d u=\left(2 a_{0} u+a_{1}\right) d u, \quad \frac{E_{v}}{E^{3 / 2}} d v=\left(2 a_{0} v+a_{2}\right) d v .
$$


From (45) we obtain

$$
E=\frac{4}{\left[a_{0}\left(u^{2}+v^{2}\right)+a_{1} u+a_{2} v+a_{3}\right]^{2}},
$$

where $a_{3}$ is another real constant.

The Gaussian curvature of $S$ is given by

$$
K=-\frac{1}{2 E} \Delta \log E\left(^{8}\right) \text {. }
$$

From (46) and (47) it follows that

$$
K=\frac{4 a_{0} a_{3}-a_{1}^{2}-a_{2}^{2}}{4},
$$

which implies that $a_{0} \neq 0$, since $S$ is on a sphere of finite non-null radius. Since (9) holds, it follows from (7) and (46) that the first quadratic form of $S$ may be written as follows:

$$
d s^{2}=\frac{\alpha^{2}\left(d u^{2}+d v^{2}\right)}{\left[\left(u+\frac{a_{1}}{2 a_{0}}\right)^{2}+\left(v+\frac{a_{2}}{2 a_{0}}\right)^{2}+\beta^{2}\right]^{2}},
$$

where

$$
\alpha=\left|2 / a_{0}\right|, \quad \beta=\left(K / a_{0}^{2}\right)^{1 / 2} .
$$

Therefore the first quadratic form of $S$ has the representation (15); hence it follows from Lemma 1 that all circles in $D$ are mapped on circles on $S$ by the functions (6). This is a contradiction of the hypothesis. Hence (21) holds for some points $\left(u_{0}, v_{0}\right)$ in $D$.

Sufficiency. By setting $m=3$ in (12), we obtain

$$
\sum_{j=1}^{3}\left[\int_{C_{r}} x_{j}(u, v) d z\right]^{2}=-\pi^{2} r^{4} \sum_{j=1}^{3}\left(\lambda x_{j}\right)^{2}-\frac{\pi^{2} r^{6}}{4} \sum_{j=1}^{3} \lambda x_{j} \Delta \lambda x_{j}+o\left(r^{6}\right),
$$

where $C_{r}$ is the circle in $D$ with center at $\left(u_{0}, v_{0}\right)$ and radius $r$, and where the partial derivatives are evaluated at $\left(u_{0}, v_{0}\right)$. From (20) and (48) it follows that (9) and

$$
\sum_{j=1}^{3} \lambda x_{j} \Delta \lambda x_{j}=0
$$

hold. Operating on (9) with the operator $\bar{\lambda}$ we obtain

(8) E. F. Beckenbach and T. Rad6, Subharmonic functions and surfaces of negative curvature, these Transactions, vol. 35 (1933), pp. 662-674. 


$$
\sum_{j=1}^{3} \lambda x_{j} \Delta x_{j}=0
$$

Operating on (50) with $\lambda$, and applying (49) to the result, we obtain

$$
\sum_{j=1}^{3} \lambda^{2} x_{j} \Delta x_{j}=0
$$

The four real linear homogeneous equations in $\Delta x_{j}, j=1,2,3$, implied by (50) and (51) are

$$
\begin{array}{rlrl}
\sum_{j=1}^{3} x_{j, u} \Delta x_{j} & =0, & \sum_{j=1}^{3} x_{j, v} \Delta x_{j}=0, \\
\sum_{j=1}^{3}\left(x_{j, u u}-x_{j, v v}\right) \Delta x_{j} & =0 & \sum_{j=1}^{3}\left(x_{j, u v}\right) \Delta x_{j} & =0 .
\end{array}
$$

One solution of $(52)$ is

$$
\Delta x_{j} \equiv 0,
$$

$$
j=1,2,3,
$$

which, by (9) and the theorem of Weierstrass, implies that the functions (6) map $D$ isothermically on a minimal surface. From (9), (12) and (53) it follows that

$$
\sum_{j=1}^{3}\left[\int_{C} x_{j}(u, v) d z\right]^{2}=0
$$

for all circles $C$ in $D$. Therefore (37) holds. This leads to a contradiction of (21); hence the functions (6) do not satisfy (53). It follows that the given functions, which satisfy (52) and do not satisfy (53), must be functions for which the rank of the matrix

$$
\left\|\begin{array}{ccc}
x_{1, u} & x_{2, u} & x_{3, u} \\
x_{1, v} & x_{2, v} & x_{3, v} \\
x_{1, u v} & x_{2, u v} & x_{3, u v} \\
x_{1, u u}-x_{1, v v} & x_{2, u u}-x_{2, v v} & x_{3, u u}-x_{3, v v}
\end{array}\right\|
$$

is less than three; hence, from the definitions of $e, f, g$, we obtain $e=g, f=0$, which, with (9), imply that the functions (6) are the coordinate functions of a surface $S$ that lies on a sphere $\delta\left({ }^{9}\right)$ of finite $\left({ }^{10}\right)$ non-null radius.

(9) It is easily seen that the hypothesis of Theorem 3 on page 98 , Graustein, op. cit., is satisfied.

(10) If the surface $S$ were a plane surface, then the functions (6) would be the isothermic coordinate functions of a minimal surface. We have already considered functions (6) that satisfy (9) and (53). 
Suppose that the functions (6) map $D$ isothermically on the spherical surface $S$ such that circles are mapped on circles $\left({ }^{11}\right)$. Then the map of an arbitrary fixed circle $C^{\prime}$ in $D$ is a circle, $C^{*}$, on $S$. Project $S$ stereographically on the plane $p$ of $C^{*}$ and let the map of $S$ on $p$ be $D^{*}$. It follows that $D$ has been mapped isothermically on the plane surface $D^{*}$. By the theorem of Weierstrass, the mapping functions form a triple of conjugate harmonic functions,

$$
y_{j}=y_{j}(u, v), \quad j=1,2,3,
$$

where (9) and $\Delta y_{j} \equiv 0, j=1,2,3$, hold. Just as for (54) it follows that

$$
\sum_{j=1}^{3}\left[\int_{C^{\prime}} y_{j}(u, v) d z\right]^{2}=0
$$

for the arbitrary fixed circle $C^{\prime}$ in $D$. But, for $(u, v)$ on $C^{\prime}$,

$$
x_{j}(u, v)=y_{j}(u, v), \quad j=1,2,3,
$$

which, with (55) implies that (54) holds. But this leads to a contradiction of (21). Therefore the functions (6) map $D$ isothermically on the spherical surface $S$ such that no non-null circle in $D$ is mapped on a circle on $S$.

\section{ChaRACTERIZATION OF MINIMAL SURFACES IN ISOTHERMiC REPRESENTATION AND OF THOSE ISOTHERMIC SPHERICAL MAPS THAT MAP CIRCLES ON CIRCLES}

THEOREM 3. If the functions (6) have continuous partial derivatives of the third order in a simply connected domain $D$, then a necessary and sufficient condition that they either (1) be the coordinate functions of a minimal surface in isothermic representation, or (2) map $D$ isothermically on a surface $S$ that lies on a sphere of finite non-null radius such that circles are mapped on circles, is that for each circle $C$ in $D$

$$
\sum_{j=1}^{3}\left[\int_{C} x_{j}(u, v) d z\right]^{2}=0 .
$$

Necessity. We have already shown that if the functions (6) are the coordinate functions of a minimal surface in isothermic representation, then (56), i.e., (54) holds. We have also shown that if the functions (6) map $D$ isothermically on a surface $S$ that lies on a sphere with finite non-null radius such that circles are mapped on circles, then (56) holds $\left({ }^{12}\right)$.

Sufficiency. If (56) holds, then (37) holds. It follows that (9) and (49) hold; therefore, as in the proof of Theorem 2, we obtain the system of equa-

(11) We have already indicated that if one non-null circle in $D$ is mapped on a circle on $S$, then all circles in $D$ are mapped on circles on $S$. See the last paragraph of the first part of the proof of Lemma 1.

(12) See the latter half of the proof of Theorem 2. 
tions (52). Then, as before, it follows that the functions (6) either are the coordinate functions of a minimal surface in isothermic representation or they map $D$ isothermically on a surface $S$ that lies on a sphere with finite non-null radius $\left({ }^{13}\right)$. Here we have made use of the results of the second part of the proof of Theorem 2. But in the first part of the proof of Theorem 2 we have shown that if $S$ lies on a sphere of finite non-null radius, (37) implies that the first quadratic form of $S$ has the representation (15); hence, by Lemma 1, all circles in $D$ are mapped on circles on $S$.

Corollary 1. If the functions (6) have continuous partial derivatives of the third order in a simply connected domain $D$, then a necessary and sufficient condition that

$$
\sum_{j=1}^{3}\left[\int_{C} x_{j}(u, v) d z\right]^{2}=0
$$

hold for each circle $C$ in $D$ is that for each point $\left(u_{0}, v_{0}\right)$ in $D$,

$$
\sum_{j=1}^{3}\left[\int_{C_{r}} x_{j}(u, v) d z\right]^{2}=o\left(r^{8}\right)
$$

hold, where $C_{r}$ is the circle in $D$ with center $\left(u_{0}, v_{0}\right)$ and radius $r$.

CoROLlaRY 2. If the functions (6) have continuous partial derivatives of the third order in a simply connected domain $D$, then a necessary and sufficient condition that the functions (6) either map $D$ isothermically on a minimal surface, or map $D$ isothermically on a surface $S$ that lies on a sphere with finite non-null radius such that circles are mapped on circles, is that for each point $\left(u_{0}, v_{0}\right)$ in $D$,

$$
\sum_{j=1}^{3}\left[\int_{C_{r}} x_{j}(u, v) d z\right]^{2}=o\left(r^{8}\right)
$$

hold, where $C_{r}$ is the circle in $D$ with center at $\left(u_{0}, v_{0}\right)$ and radius $r$.

\section{Characterization of isothermic Plane maps}

LEMMA 2. Let the function $g_{1}(z)$ be schlicht $\left({ }^{14}\right)$, and the function $g_{2}(z)$ analytic, in the circle $|z|<\rho$. If $\delta$ is an arbitrary positive number, $0<\delta<\rho$, then there exists a positive number $\epsilon=\epsilon(\delta)$ such that the function

$$
f(z) \equiv g_{1}(z)+\eta g_{2}(z)
$$

is schlicht in the circle $|z| \leqq \rho-\delta$, for each $\eta$ such that $|\eta|<\epsilon$.

Proof. If the lemma does not hold, then there exists a positive number $\delta_{0}$,

(13) If the sphere were either a point-sphere or a plane, then the functions (6) would form a triple of conjugate harmonic functions.

(14) The analytic function $g_{1}(z)$ is said to be schlicht in $D$ if $g_{1}\left(z_{1}\right)=g_{1}\left(z_{2}\right)$ implies $z_{1}=z_{2}$, where $z_{1}$ and $z_{2}$ are points of $D$. 
two points, $z_{0}^{\prime}$ and $z_{0}^{\prime \prime}$, and a triple of sequences

$$
\begin{gathered}
\eta_{1}, \eta_{2}, \cdots, \\
z_{1}^{\prime}, z_{2}^{\prime}, \cdots, \quad z_{1}^{\prime \prime}, z_{2}^{\prime \prime}, \cdots, \quad z_{n}^{\prime} \neq z_{n}^{\prime \prime}, n=1,2, \cdots,
\end{gathered}
$$

with the properties

$$
\begin{array}{cc}
\lim _{n \rightarrow \infty} \eta_{n}=0, & \\
\lim _{n \rightarrow \infty} z_{n}^{\prime}=z_{0}^{\prime}, \quad\left|z_{n}^{\prime}\right| \leqq \rho-\delta_{0}, & n=0,1,2, \cdots, \\
\lim _{n \rightarrow \infty} z_{n}^{\prime \prime}=z_{0}^{\prime \prime}, \quad\left|z_{n}^{\prime \prime}\right| \leqq \rho-\delta_{0}, & n=0,1,2, \cdots, \\
f_{n}\left(z_{n}^{\prime}\right)=f_{n}\left(z_{n}^{\prime \prime}\right), & n=1,2, \cdots,
\end{array}
$$

where

$$
f_{n}(z) \equiv g_{1}(z)+\eta_{n} g_{2}(z), \quad n=1,2, \cdots .
$$

It follows immediately that

$$
g_{1}\left(z_{0}^{\prime}\right)=g_{1}\left(z_{0}^{\prime \prime}\right) \text {. }
$$

But since $g_{1}(z)$ is schlicht in the circle $|z|<\rho$, it follows that $z_{0}^{\prime}=z_{0}^{\prime \prime}=z_{0}$. Moreover, we obtain

$$
\lim _{n \rightarrow \infty} \frac{f_{n}\left(z_{n}^{\prime}\right)-f_{n}\left(z_{n}^{\prime \prime}\right)}{z_{n}^{\prime}-z_{n}^{\prime \prime}}=\frac{d g_{1}\left(z_{0}\right)}{d z}=0\left({ }^{15}\right) .
$$

Since $\left|z_{0}\right| \leqq \rho-\delta_{0},(58)$ implies that $g_{1}(z)$ is not schlicht in the circle $|z|<\rho$. From this contradiction of the hypothesis, it follows that there exists an $\epsilon_{0}>0$ such that the function (57) is schlicht, for each $\eta$ satisfying $|\eta|<\epsilon_{0}$, in $|z| \leqq \rho-\delta_{0}$.

THEOREM 4. If the functions (6) have continuous partial derivatives of the third order in a simply connected domain $D$, then a necessary and sufficient condition that they map $D$ isothermically on a plane surface is that for all closed rectifiable Jordan curves $\gamma$ lying in $D$

$$
\sum_{j=1}^{3}\left[\int_{\gamma} x_{j}(u, v) d z\right]^{2}=0 .
$$

(15) Since $g_{1}(z)$ is analytic in the closed region defined by $|z| \leqq \rho-\delta_{0}$, for any arbitrary positive number $\delta^{\prime}$ there exists a positive number $\epsilon^{\prime}$, which is independent of $z$ in the region $|z| \leqq \rho-\delta_{0}$, for which

$$
\left|\frac{g_{1}\left(z^{\prime}\right)-g_{1}\left(z^{\prime \prime}\right)}{z^{\prime}-z^{\prime \prime}}-\frac{d}{d z} g_{1}(z)\right|<\delta^{\prime}
$$

for all $z^{\prime}, z^{\prime \prime}$ and $z$ in the region $|z| \leqq \rho-\delta_{0}$ that satisfy the inequalities $\left|z-z^{\prime}\right|<\epsilon^{\prime},\left|z-z^{\prime \prime}\right|<\epsilon^{\prime}$, $z^{\prime} \neq z^{\prime \prime}$. 
Necessity. If the functions (6) map $D$ isothermically on a surface $S$ that lies on a plane $p$, we make a rigid transformation in the $x_{1}, x_{2}, x_{3}$-space such that $p$ coincides with the plane $x_{3}^{\prime}=0$ and the positive normal at the image of an arbitrary fixed point $\left(u_{0}, v_{0}\right)$ of $D$ coincides with the positive $x_{3}^{\prime}$-axis. Let this rigid transformation have the representation

$$
x_{j}^{\prime}=\sum_{k=1}^{3} \lambda_{k j} x_{k}+a_{j}, \quad j=1,2,3,
$$

where

$$
\begin{aligned}
\sum_{k=1}^{3} \lambda_{s k} \lambda_{j k} & =0, s \neq j, \\
& =1, s=j, \quad s, j=1,2,3,
\end{aligned}
$$

and where the $a_{j}, j=1,2,3$, are real constants. Therefore the functions

$$
\begin{gathered}
x_{j}^{\prime}(u, v)=\sum_{k=1}^{3} \lambda_{k j}\left[x_{k}(u, v)-x_{k}\left(u_{0}, v_{0}\right)\right], \quad j=1,2,3, \\
x_{3}^{\prime}(u, v) \equiv 0,
\end{gathered}
$$

map $D$ isothermically on a plane surface, and hence, by the theorem of Weierstrass, they form a triple of conjugate harmonic functions. Since it follows from (8), (9), (61) and (62) that (3) holds for the functions $x_{1}^{\prime}(u, v)$ and $x_{2}^{\prime}(u, v)$, the functions $x_{1}^{\prime}(u, v)$ and $x_{2}^{\prime}(u, v)$ are a couple of conjugate harmonic functions. Hence by Cauchy's theorem, analogous to (5), and (62), it follows that

$$
\sum_{j=1}^{3}\left[\int_{\gamma} x_{j}^{\prime}(u, v) d z\right]^{2}=0,
$$

which, with (4), (60) and (61), yields (59).

Sufficiency. If (59) holds for all closed rectifiable Jordan curves $\gamma$ lying in $D$, then, by Theorem 3 , the functions (6) either are the coordinate functions of a minimal surface in isothermic representation, or map $D$ isothermically on a surface $S$ that lies on a sphere of finite non-null radius such that circles are mapped on circles.

Part I. We first consider the case when the functions (6) are the coordinate functions of a minimal surface in isothermic representation. Let $\left(u_{0}, v_{0}\right)$ be an arbitrary point in $D$ and let the arbitrary fixed circle $C_{0}$ in $D$ have its center at $\left(u_{0}, v_{0}\right)$. If $\gamma$ is an arbitrary closed rectifiable Jordan curve lying in $C_{0}$, which contains $\left(u_{0}, v_{0}\right)$ in its interior, then the interior of $\gamma$ can be mapped conformally on the interior of the circle $C: s^{2}+t^{2}=\rho^{2}$ in the $s, t$-plane such that the image of $\left(u_{0}, v_{0}\right)$ is the center of $C$. The expansion of the inverse of this mapping function has the representation 


$$
z=h(w)=z_{0}+\sum_{m=1}^{\infty} b_{m} w^{m}
$$

where

$$
w=s+i t, \quad z_{0}=u_{0}+i v_{0} .
$$

Since the functions (6) are the isothermic coordinate functions of a minimal surface, it follows, from the theorem of Weierstrass, that these functions may be written in the form

$$
x_{j}(u, v)=\frac{1}{2}\left[\Phi_{j}(z)+\overline{\Phi_{j}(z)}\right], \quad j=1,2,3,
$$

where $\Phi_{j}(z)$ is a function which is analytic in $D$ and where $\overline{\Phi_{j}(z)}$ is its conjugate function. We may write

$$
\Phi_{j}(z)=\sum_{n=0}^{\infty} a_{j, n}\left(z-z_{0}\right)^{n}, \quad j=1,2,3 ;
$$

then

$$
\overline{\Phi_{j}(z)}=\sum_{n=0}^{\infty} \bar{a}_{j, n}\left(\overline{\left.z-z_{0}\right)^{n}}, \quad j=1,2,3,\right.
$$

where the series for $\Phi_{j}(z)$ and $\overline{\Phi_{j}(z)}$ are absolutely convergent in the interior of and on $C_{0}$. Therefore

$$
\overline{\Phi_{j}(z)}=\bar{a}_{j, 0}+\sum_{n=1}^{\infty} \bar{a}_{j, n} \sum_{m=1}^{\infty} P_{n, m} \bar{w}^{m}, \quad j=1,2,3,
$$

where

$$
\begin{array}{r}
P_{n, m}=\sum_{k_{l}} b_{k_{1}} \cdots \bar{b}_{k_{n}}, n \leqq m, k_{1}+\cdots+k_{n}=m, k_{l} \geqq 1, l=1,2, \cdots, n, \\
P_{n, m}=0, \\
n>m .
\end{array}
$$

If $C_{R}$ is the circle concentric with $C$ and of radius $R, 0<R<\rho$, then the function (63) maps the interior of $C_{R}$ conformally on the interior of a closed rectifiable Jordan curve $\gamma_{R}$ that lies in the interior of $\gamma$. After we have set $w=R e^{i \phi}$ in (63) and (65), it follows from (4), (63), (64), (65) and (66) that

$$
\sum_{j=1}^{3}\left[\int_{\gamma_{R}} x_{j}(u, v) d z\right]^{2}=-\pi^{2} \sum_{t=2}^{\infty} \sum_{m=1}^{t-1} \sum_{n=1}^{m} \sum_{s=1}^{t-m} A_{n, s} B_{n, m} B_{s, t-m} R^{2 t},
$$

which, with (59), implies

$$
\sum_{t=2}^{\infty} \sum_{m=1}^{t-1} \sum_{n=1}^{m} \sum_{s=1}^{t-m} A_{n, 8} B_{n, m} B_{s, t-m} R^{2 t}=0
$$

where 


$$
B_{n, m}=m b_{m} P_{n, m},
$$

$$
n, m=1,2, \cdots
$$

and where

$$
A_{n, s}=A_{s, n}=\sum_{j=1}^{3} \bar{a}_{j, n} \bar{a}_{j, s}, \quad n, s=1,2, \cdots
$$

But the circle $C_{R}$ is an arbitrary circle concentric with and interior to the circle $C$; therefore the relation (67) is independent of $R, 0<R<\rho$. Hence

$$
\sum_{m=1}^{t-1} \sum_{n=1}^{m} \sum_{s=1}^{t-m} A_{n, s} B_{n, m} B_{s, t-m}=0, \quad t=2,3, \ldots
$$

From (70) we obtain, by an induction,

$$
A_{n, 8}=0 \text {, }
$$$$
n, s=1,2, \cdots
$$

For, when $t=2$ in (70) we obtain, by (66) and (68),

$$
A_{1,1} b_{1}^{2} b_{1}^{2}=0 \text {. }
$$

But the function (63) maps the interior of $C$ on the interior of $\gamma$ in a one-toone manner $\left({ }^{16}\right)$. Therefore $b_{1} \neq 0$. Hence it follows from (72) that

$$
A_{1,1}=0 \text {. }
$$

Now suppose that

$$
A_{n, 8}=0, \quad 2 \leqq n+s \leqq p-1, p \geqq 3,
$$

where $n, s$ and $p$ are positive integers. We shall show that

$$
A_{n, 8}=0 \text {, }
$$$$
n+s=p
$$

For $t=p,(70)$ yields, by (66), (68), (74) and the fact that $b_{1} \neq 0$,

$$
\sum_{k=1}^{p-1} k(p-k) b_{k} b_{p-k} A_{k, p-k}=0 .
$$

By Lemma 2, for a fixed $R, 0<R<\rho$, the functions

$$
G_{q}(w)=h(w)+\eta_{q} w^{q}=z_{0}+\sum_{m=1}^{\infty} b_{m} w^{m}+\eta_{q} w^{q}, \quad q=1,2, \cdots, p-1,
$$

are schlicht in the circle $|w| \leqq R$, provided

$$
\left|\eta_{q}\right|<\epsilon_{q}, \quad q=1,2, \cdots, p-1,
$$

where $\epsilon_{q}$ is a positive constant whose existence was established in Lemma 2.

(16) This is a consequence of Darboux's theorem. See W. F. Osgood, Functions of a Complex Variable, p. 167. 
We further restrict $\eta_{q}$ to satisfy the relation

$$
R^{q}\left|\eta_{q}\right|<\delta,
$$

for $q=1,2, \cdots, p-1$, where $\delta$ is the distance between $\gamma_{R}$ and $C_{0}$, in order that the map $\gamma_{q, R}$ of $C_{R}$ by (77) shall lie inside $C_{0}$. Moreover, $\left(u_{0}, v_{0}\right)$ is inside $\gamma_{q, R}$. Hence if

$$
0<\left|\eta_{q}\right|<d_{q}=\min \left[\epsilon_{q}, \frac{\delta}{R^{q}}\right], \quad q=1,2, \cdots, p-1,
$$

then we obtain the following result, which is analogous to (76), for the functions (77):

$$
\begin{aligned}
& \sum_{k=1}^{p-1} k(p-k) b_{k} b_{p-k} A_{k, p-k}+2 q(p-q) b_{p-q} \eta_{q} A_{q, p-q}=0, \\
& \sum_{k=1}^{p-1} k(p-k) b_{k} b_{p-k} A_{k, p-k}+q^{2}\left(\eta_{q}^{2}+2 \eta_{q} b_{q}\right) A_{q, q}=0,
\end{aligned}
$$

where (79a) holds for all $q, 1 \leqq q \leqq p-1$, except $q=p / 2$, and where (79b) holds for $q=p / 2\left({ }^{17}\right)$. From (76) and (79) we obtain

$$
\begin{aligned}
2 q(p-q) b_{p-q} \eta_{q} A_{q, p-q} & =0, \\
q^{2}\left(\eta_{q}^{2}+2 \eta_{q} b_{q}\right) A_{q, q} & =0,
\end{aligned}
$$

where (80a) holds for all $q, 1 \leqq q \leqq p-1$, except $q=p / 2$, and where (80b) holds for $q=p / 2$.

Since $\eta_{q}$ is an arbitrary constant which is subject only to the restriction (78), it follows from the relation (80b) that

$$
A_{q, q}=0, \quad q=p / 2,
$$

provided $p$ is even. If $q_{1}$ is a fixed positive integer, $1 \leqq q_{1} \leqq p-1$, and $q_{1} \neq p / 2$, and if $b_{p-q_{1}} \neq 0$, then it follows from (80a) that

$$
A_{q_{1}, p-q_{1}}=0 \text {. }
$$

If $q_{2}$ is a fixed positive integer, $1 \leqq q_{2} \leqq p-1$ and $q_{2} \neq p / 2$, and if $b_{p-q_{2}}=0$, then we consider the function

$$
h_{1}(w)=h(w)+\eta_{p-q_{2}}^{\prime} w^{p-q_{2}},
$$

where $\eta_{p-q_{2}}^{\prime}$ is a fixed constant,

$$
0<\left|\eta_{p-q_{2}}^{\prime}\right|<d_{p-q_{2}}
$$

(17) When we state "except for $q=p / 2$," we mean that if $p$ is an even integer, then $q$ does not take on the integral value $p / 2$. 
The function (83) is schlicht in the circle $|w| \leqq R\left({ }^{18}\right)$, and maps $C_{R}$ on a closed rectifiable Jordan $\gamma_{R}{ }^{\prime}$ that lies inside $C_{0}$. Moreover, $\left(u_{0}, v_{0}\right)$ is inside $\gamma_{R}^{\prime}$. From (63) and (83) we obtain

$$
h_{1}(w)=z_{0}+\sum_{m=1}^{\infty} c_{m} w^{m},
$$

where

$$
c_{m}=b_{m}, \quad m \neq p-q_{2} ; \quad c_{p-q_{2}}=\eta_{p-q_{2}}^{\prime} .
$$

If we apply (84) as we have applied (63) in the earlier part of this proof of Theorem 4 , then we obtain the following result analogous to (80a):

$$
2 q_{2}\left(p-q_{2}\right) \eta_{p-q_{2}}^{\prime} \eta_{q_{2}}^{\prime \prime} A_{q_{2}, p-q_{2}}=0,
$$

where $\eta_{q_{2}}^{\prime \prime}$ is an arbitrary constant,

$$
0<\left|\eta_{q_{2}}^{\prime \prime}\right|<d_{q_{2}}^{\prime} \text {. }
$$

Here the constant $d_{q_{2}}^{\prime}$ is in the same relation to $h_{1}(w)$ as $d_{q_{2}}$ is to $h(w)$. Since $\eta_{p-q_{2}}^{\prime} \neq 0$ and $q_{2} \neq p / 2$, it follows from (85) and (86) that

$$
A_{q_{2}, p-q_{2}}=0 \text {. }
$$

From (81), (82) and (87) it follows that (75) holds. Since (73) holds, our induction is now complete.

From (69), (71) and the substitution

$$
a_{j, m}=\alpha_{j, m}-i \beta_{j, m}, \quad j=1,2,3, m=0,1, \cdots,
$$

we get the following relations:

$$
\begin{aligned}
& \sum_{j=1}^{3}\left(\alpha_{j, n} \alpha_{j, 8}-\beta_{j, n} \beta_{j, 8}\right)=0, \\
& \sum_{j=1}^{3}\left(\alpha_{j, n} \beta_{j, 8}+\alpha_{j, s} \beta_{j, n}\right)=0,
\end{aligned}
$$

here $\alpha_{j, 0}, \alpha_{j, m}$ and $\beta_{j, m}, m=1,2, \cdots$, are the Fourier coefficients in the expansion of $x_{j}(u, v), j=1,2,3$, about the point $\left(u_{0}, v_{0}\right)$.

We transform the axes in the $x_{1}, x_{2}, x_{3}$-space such that the new origin is at the image of $\left(u_{0}, v_{0}\right)$, the plane $x_{3}^{\prime}=0$ is tangent to the surface there, and the positive normal to the surface there coincides with the positive $x_{3}^{\prime}$-axis. This transformation is given by (60) and (61), where

(18) This holds because $\left|\eta_{p-q_{2}}^{\prime}\right|<d_{p-q_{2}}$, where $d_{p-q_{2}}$ was defined in (78).

$\left.{ }^{19}\right)$ Compare with J. W. Hahn and E. F. Beckenbach, Triples of conjugate harmonic functions and minimal surfaces, Duke Mathematical Journal, vol. 2 (1936), Lemma 1, p. 699, and footnote, p. 700. 


$$
a_{j}=-\sum_{k=1}^{3} \lambda_{k j} x_{k}\left(u_{0}, v_{0}\right)
$$

$$
j=1,2,3 \text {. }
$$

The relations (88) are invariant under this rigid transformation. For, since the functions (6) form a triple of conjugate harmonic functions, it follows that the new coordinate functions,

$$
x_{j}^{\prime}(u, v)=\sum_{k=1}^{3} \lambda_{k j}\left[x_{k}(u, v)-x_{k}\left(u_{0}, v_{0}\right)\right], \quad j=1,2,3,
$$

form a triple of conjugate harmonic functions. Hence we may write, in terms of polar coordinates,

$$
x_{j}^{\prime}(u, v)=\alpha_{j, 0}^{\prime}+\sum_{m=1}^{\infty} r^{m}\left(\alpha_{j, m}^{\prime} \cos m \theta+\beta_{j, m}^{\prime} \sin m \theta\right), \quad j=1,2,3,
$$

where

$$
\alpha_{j, m}^{\prime}=\sum_{k=1}^{3} \lambda_{k j} \alpha_{k, m}, \quad \beta_{j, m}^{\prime}=\sum_{k=1}^{3} \lambda_{k j} \beta_{k, m}, \quad m=1,2, \cdots, j=1,2,3 .
$$

From (61), (88) and (90) we obtain

$$
\begin{array}{ll}
\sum_{j=1}^{3}\left(\alpha_{j, n}^{\prime} \alpha_{j, 8}^{\prime}-\beta_{j, n}^{\prime} \beta_{j, 8}^{\prime}\right)=0, & \\
\sum_{j=1}^{3}\left(\alpha_{j, n}^{\prime} \beta_{j, 8}^{\prime}+\alpha_{j, 8}^{\prime} \beta_{j, n}^{\prime}\right)=0, & n, s \geqq 1 .
\end{array}
$$

Therefore the relations (88) are invariant under a rigid transformation. show

To prove that the functions (6) define a plane surface, it is sufficient to

$$
\alpha_{3,0}^{\prime}=\alpha_{3, m}^{\prime}=\beta_{3, m}^{\prime}=0,
$$

$$
m=1,2, \cdots
$$

Since $x_{j}^{\prime}\left(u_{0}, v_{0}\right)=0, j=1,2,3$, it follows that

$$
\alpha_{j, 0}^{\prime}=0,
$$

$$
j=1,2,3 \text {. }
$$

Let $t$ be the positive integer for which

$$
\begin{aligned}
& \sum_{j=1}^{3} \alpha_{j, m}^{\prime 2}=0, \quad m=0,1, \cdots, t-1, \\
& \sum_{j=1}^{3} \alpha_{j, t}^{\prime 2} \neq 0\left({ }^{20}\right) .
\end{aligned}
$$

${ }^{(20)}$ If $\sum_{j=1}^{3} \alpha_{j, m}^{2}=0$ for all positive $m$, then it follows, from the first relation in (91), that $\sum_{j=1}^{3} \beta_{j, m}^{\prime 2}=0$ for all positive $m$; and therefore (92) holds. 
From (89) we obtain

$$
\begin{aligned}
& \alpha_{2, t}^{\prime} \beta_{3, t}^{\prime}-\alpha_{3, t}^{\prime} \beta_{2, t}^{\prime}=0 \\
& \alpha_{3, t}^{\prime} \beta_{1, t}^{\prime}-\alpha_{1, t}^{\prime} \beta_{3, t}^{\prime}=0, \\
& \left.\alpha_{1, t}^{\prime 2}+\alpha_{2, t}^{\prime 2}+\alpha_{3, t}^{\prime 2}=\alpha_{1, t}^{\prime} \beta_{2, t}^{\prime}-\alpha_{2, t}^{\prime} \beta_{1, t}^{\prime}{ }^{21}\right),
\end{aligned}
$$

since the positive normal to $S$ at the point $(0,0,0)$ coincides with the positive $x_{3}^{\prime}$-axis. Equations (95a) and (95b) are linear and homogeneous in $\alpha_{3, t}^{\prime}$ and $\beta_{3, t}^{\prime}$; since by (94b) and (95c) their determinant of coefficients is not zero, it follows that

$$
\alpha_{3, t}^{\prime}=\beta_{3, t}^{\prime}=0 \text {. }
$$

From (91), for $n=s=t,(94 \mathrm{~b})$ and (96) we obtain

$$
\begin{aligned}
& \alpha_{1, t}^{\prime 2}+\alpha_{2, t}^{\prime 2}={\beta_{1, t}^{\prime 2}}^{\prime 2}+{\beta_{2, t}^{\prime 2}}^{\prime 2} \neq 0, \\
& \alpha_{1, t}^{\prime} \beta_{1, t}^{\prime}+\alpha_{2, t}^{\prime} \beta_{2, t}^{\prime}=0,
\end{aligned}
$$

which, with (95c) and (96), imply

$$
\alpha_{1, t}^{\prime}=\beta_{2, t}^{\prime}, \quad \alpha_{2, t}^{\prime}=-\beta_{1, t}^{\prime} .
$$

From (91), for $s=t \leqq n,(96)$ and (98) we obtain

$$
\begin{aligned}
& \alpha_{1, n}^{\prime} \alpha_{1, t}^{\prime}+\alpha_{2, n}^{\prime} \alpha_{2, t}^{\prime}+\alpha_{2, t}^{\prime} \beta_{1, n}^{\prime}-\alpha_{1, t}^{\prime} \beta_{2, n}^{\prime}=0, \\
& -\alpha_{1, n}^{\prime} \alpha_{2, t}^{\prime}+\alpha_{2, n}^{\prime} \alpha_{1, t}^{\prime}+\alpha_{2, t}^{\prime} \beta_{2, n}^{\prime}+\alpha_{1, t}^{\prime} \beta_{1, n}^{\prime}=0, \quad n \geqq t .
\end{aligned}
$$

If we eliminate first $\alpha_{2, n}^{\prime}$ and then $\beta_{2, n}^{\prime}$ from these last two relations, then we obtain two other relations which, with the first relation in (97), imply

$$
\alpha_{1, n}^{\prime}=\beta_{2, n}^{\prime}, \quad \alpha_{2, n}^{\prime}=-\beta_{1, n}^{\prime}, \quad n \geqq t .
$$

From (91), for $n=s \geqq t$, and (99) we obtain

$$
\alpha_{3, n}^{\prime} \beta_{3, n}^{\prime}=0, \quad \alpha_{3, n}^{\prime 2}={\beta_{3, n}^{\prime 2},}_{2}, \quad n t,
$$

from which it follows that

$$
\alpha_{3, n}^{\prime}=\beta_{3, n}^{\prime}=0,
$$$$
n \geqq t \text {. }
$$

From (91), for $n=s<t$, (94a) and (100), we obtain (92).

(21) If $z-z_{0}=r(\cos \theta+i \sin \theta)$, then for $r$ sufficiently small the components of the unit normal vector to the surface are $\zeta_{j}=\zeta_{j}(u, v), j=1,2,3, \zeta_{j}(u, v)=\left[\left(\alpha_{k, t}^{\prime}, \beta_{l, t}^{\prime}-\alpha_{l, t}^{\prime} \beta_{k, t}^{\prime}\right) / \sum_{j=1}^{8} \alpha_{i, t}^{\prime 2}\right]+O(r)$, where $O(r)$ denotes a quantity $\psi(r)$ (not always the same quantity) such that $|\psi(r) / r|$ is bounded. Note that $\zeta_{1}=0, \zeta_{2}=0, \zeta_{3}=1$ at $(0,0,0)$. 
COROLLARY. If the functions (6) are harmonic in a simply connected domain $D$, such that their Fourier expansions about the fixed point $\left(u_{0}, v_{0}\right)$ of $D$ are

$$
x_{j}(u, v)=\alpha_{j, 0}+\sum_{m=1}^{\infty} r^{m}\left(\alpha_{j, m} \cos m \theta+\beta_{j, m} \sin m \theta\right), \quad j=1,2,3,
$$

and if

$$
\sum_{j=1}^{3}\left(\alpha_{j, n} \alpha_{j, 8}-\beta_{j, n} \beta_{j, 8}\right)=0, \quad \sum_{j=1}^{3}\left(\alpha_{j, n} \beta_{j, 8}+\alpha_{j, 8} \beta_{j, n}\right)=0, \quad n, s \geqq 1,
$$

then the functions (6) are the isothermic coordinate functions of a plane surface.

Part II. We now consider the case when the functions (6) map $D$ isothermically on a surface $S$ that lies on a sphere $\mathcal{S}$, whose radius $a$ is finite and nonnull, such that circles are mapped on circles. Since the functions (6) have the representation (18), it follows that they may be continued isothermically to map the whole $u, v$-plane isothermically on $S$ such that circles are mapped on circles. Let the point $P$ on $S$ correspond to the point $z=\infty$, and let $p$ be the equatorial plane corresponding to $P$ as a pole. Then the stereographic projection of $S$ on $p$, with $P$ as pole, induces an isothermic map of the $u, v$-plane on $p$ such that circles are mapped on circles, and such that the point at infinity in the $u, v$-plane corresponds to the point at infinity in the plane $p$. Let us take the center $M$ of $\mathcal{S}$ as the origin in a system of coordinates on $p$, such that the positive $s$-axis, the positive $t$-axis, and the ray $M P$, in that order, have the same disposition as the coordinate axes $\left(x_{1}, x_{2}, x_{3}\right)$. The mapping function must have one of the following representations:

$$
w=s+i t=f(z)=\alpha z+\beta,
$$

or

$$
w=s+i t=f(z)=\alpha \bar{z}+\beta,
$$

$\alpha \neq 0$,

where (101a) holds if the map of the $u, v$-plane on $p$ is directly conformal, and where (101b) holds if the map is inversely conformal.

Consider the system of axes $\left(x_{1}^{\prime}, x_{2}^{\prime}, x_{3}^{\prime}\right)$ in space, with origin at $M$, and with the positive $x_{1}^{\prime}-, x_{2}^{\prime}$ - and $x_{3}^{\prime}$-axes having the directions of the positive $s$-axis, the positive $t$-axis and the ray $M P$ respectively. There exists a rigid transformation in the $x_{1}, x_{2}, x_{3}$-space which carries the origin into the point $M$, and which carries the positive $x_{1^{-}}, x_{2}$ - and $x_{3}$-axes into the positive $x_{1}^{\prime}-, x_{2}^{\prime}$ - and $x_{3}^{\prime}$-axes respectively; this transformation has the form (60), where (61) holds. Let the new coordinate functions of $S$ be

$$
x_{j}^{\prime}=x_{j}^{\prime}(u, v), \quad j=1,2,3 .
$$

Since the functions (6) map circles on circles, the functions (102) map circles on circles; therefore, as noted in Lemma 1, the functions (102) have the form 
(18) where $f(z)$ is given by (101). Hence, since $\alpha \neq 0$ in (101), we have, for the arbitrary closed rectifiable Jordan curve $\gamma$ in $D$,

$$
\sum_{j=1}^{3}\left[\int_{\gamma} x_{j}^{\prime}(u, v) d z\right]^{2}=\frac{4 a^{4}}{\alpha^{2}} \sum_{j=1}^{3}\left[\int_{\gamma^{\prime}} \phi_{j}(s, t) d w\right]^{2},
$$

where $\gamma^{\prime}$ is the image of $\gamma$ on the plane $p$ and where

$$
\phi_{1}(s, t)=\frac{s}{a^{2}+s^{2}+t^{2}}, \quad \phi_{2}(s, t)=\frac{t}{a^{2}+s^{2}+t^{2}}, \quad \phi_{3}(s, t)=\frac{a}{a^{2}+s^{2}+t^{2}} .
$$

As it previously has been shown $\left({ }^{22}\right),(59)$ is invariant under a rigid transformation; hence, since the radius $a$ of $S$ is different from zero, it follows from (59) and (103) that

$$
\sum_{j=1}^{3}\left[\int_{\gamma^{\prime}} \phi_{j}(s, t) d w\right]^{2}=0
$$

holds. Since $\gamma$ is an arbitrary closed rectifiable Jordan curve in $D$, it follows that (104) holds for each closed rectifiable Jordan curve $\gamma^{\prime}$ in $D^{\prime}$, where $D^{\prime}$ is the map on the plane $p$ by (101).

Without any loss of generality, we may assume the line $s=0$ passes through $D^{\prime}$. Then there exists a closed rectifiable Jordan curve $\Gamma$ in $D^{\prime}$ with the following description:

(1) the vertices of $\Gamma$ have the following polar coordinates,

$$
\begin{aligned}
& A_{1}: \quad(r, \tau), \quad A_{2}:(r,-\tau), \\
& A_{3}: \quad(r+\omega,-\tau), \quad A_{4}: \quad(r+\omega, \tau), \quad 0<\tau<\pi / 2,0<r, 0<\omega ;
\end{aligned}
$$

(2) $\Gamma$ is composed of two arcs of circles and two straight-line segments: arc $A_{1} A_{2}$ is an arc of the circle $s^{2}+t^{2}=r^{2}$, arc $A_{3} A_{4}$ is an arc of the circle $s^{2}+t^{2}=(r+\omega)^{2}$, each arc subtending an angle $2 \tau$ at the origin, and $A_{2} A_{3}$ and $A_{4} A_{1}$ are on rays through the origin.

For the closed rectifiable Jordan curve $\Gamma$ we obtain

$$
\sum_{j=1}^{3}\left[\int_{\Gamma} \phi_{j}(s, t) d w\right]^{2}=-\xi \chi_{1}(r, \omega) \sin \xi-4 \chi_{2}^{2}(r, \omega) \sin ^{2} \frac{\xi}{2}, \quad \xi=2 \tau,
$$

where

$$
\begin{aligned}
\chi_{1}(r, \omega) \equiv\left[\frac{(r+\omega)^{2}}{a^{2}+(r+\omega)^{2}}-\frac{r^{2}}{a^{2}+r^{2}}\right]\left[\frac{(r+\omega)^{2}}{a^{2}+(r+\omega)^{2}}-\frac{r^{2}}{a^{2}+r^{2}}\right. \\
\left.+\log \frac{a^{2}+r^{2}}{a^{2}+(r+\omega)^{2}}\right]
\end{aligned}
$$

${ }^{(22)}$ See the first part of this proof of Theorem 4. 
$\chi_{2}(r, \omega) \equiv\left[a\left(\frac{r+\omega}{a^{2}+(r+\omega)^{2}}-\frac{r}{a^{2}+r^{2}}\right)+\arctan \frac{r}{a}-\arctan \frac{r+\omega}{a}\right]$.

Since

$$
\frac{\partial \chi_{2}}{\partial \omega}=-\frac{2 a(r+\omega)^{2}}{\left[a^{2}+(r+\omega)^{2}\right]^{2}} \not \equiv 0,
$$

it follows that $\chi_{2}(r, \omega) \not \equiv 0$. Fix $r$ and $\omega$ so that $\chi_{2}(r, \omega) \neq 0$. Since $\xi \sin \xi$ and $4 \sin ^{2} \xi / 2$ are not proportional,

$$
\xi \sin \xi=\xi^{2}-\frac{\xi^{4}}{6}+\cdots, \quad 4 \sin ^{2} \frac{\xi}{2}=\xi^{2}-\frac{\xi^{4}}{12}+\cdots,
$$

there exists a $\xi$ such that

$$
\sum_{j=1}^{3}\left[\int_{\Gamma} \phi_{j}(s, t) d w\right]^{2} \neq 0 .
$$

Now (105) is a contradiction of (104). Therefore the functions (6) do not map $D$ on a surface that lies on a sphere of finite non-null radius.

The Rice Institute,

Houston, Texas. 